\title{
Consumption of long-chain $n-3$ PUFA, $\alpha$-linolenic acid and fish is associated with the prevalence of chronic kidney disease
}

\author{
Bamini Gopinath $^{1}$, David C. Harris ${ }^{2}$, Victoria M. Flood ${ }^{3}$, George Burlutsky $^{1}$ and Paul Mitchell ${ }^{1 *} \dagger$ \\ ${ }^{1}$ Centre for Vision Research, Department of Ophthalmology and Westmead Millennium Institute, University of Sydney, \\ Sydney, NSW, Australia \\ ${ }^{2}$ Centre for Transplantation and Renal Research, Westmead Millennium Institute, University of Sydney, Sydney, NSW, \\ Australia \\ ${ }^{3}$ Faculty of Health and Behavioural Sciences, University of Wollongong, Sydney, NSW, Australia \\ (Received 7 September 2010 - Revised 5 November 2010 - Accepted 7 November 2010 - First published online 24 January 2011)
}

\section{Abstract}

Due to the anti-inflammatory properties of PUFA, it has been suggested that they may protect against kidney damage in adults. However, relatively few epidemiological studies have examined this hypothesis in human subjects. We investigated the association between dietary intakes of PUFA ( $n-3, n-6$ and $\alpha$-linolenic acid), fish and the prevalence of chronic kidney disease (CKD). A total of 2600 Blue Mountains Eye Study (1997-9) participants aged $\geq 50$ years were analysed. Dietary data were collected using a semi-quantitative FFQ, and PUFA and fish intakes were calculated. Baseline biochemistry including serum creatinine was measured. Moderate CKD was defined as an estimated glomerular filtration rate of $<60 \mathrm{ml} / \mathrm{min}$ per $1.73 \mathrm{~m}^{2}$. Participants in the highest quartile of long-chain $n$-3 PUFA intake had a significantly reduced likelihood of having CKD compared with those in the lowest quartile of intake (multivariable-adjusted OR 0.69, 95\% CI 0.49, 0.99). $\alpha$-Linolenic acid intake was positively associated with CKD (OR, per standard deviation increase in $\alpha$-linolenic acid, $1 \cdot 18,95 \%$ CI 1.05 , 1.32). Total $n$-3 PUFA or total $n-6$ PUFA were not significantly associated with CKD. The highest compared with the lowest quartile of fish consumption was associated with a reduced likelihood of CKD (OR 0.68, $95 \%$ CI 0.48, 0.97; $P$ for trend $=0 \cdot 02$ ). The present study shows that an increased dietary intake of long-chain $n-3$ PUFA and fish reduces the prevalence of CKD. Hence, a diet rich in $n$ - 3 PUFA and fish could have a role in maintaining healthy kidney function, in addition to roles of these nutrients in the prevention and modulation of other diseases.

\section{Key words: Chronic kidney disease: PUFA: n-3: Fish: Blue Mountains Eye Study}

Chronic kidney disease (CKD) is an important health problem among older adults and can lead to end-stage renal disease with its need for dialysis or transplantation for survival ${ }^{(1-3)}$. Therefore, it is important to identify risk/protective factors associated with CKD at an early kidney disease stage in the continuum, at which prevention/control of disease progression is more applicable ${ }^{(4)}$. Many cohort studies have identified hypertension, diabetes, obesity, hyperlipidaemia and smoking as risk factors for the development of CKD. It is generally hypothesised that these risk factors exert their influence by increasing vascular pathology and by direct damage to kidney structures ${ }^{(1)}$.

Due to the anti-inflammatory properties of PUFA, it has been suggested that these nutrients may protect the kidneys from damage in adults; however, very few studies have examined the putative association between PUFA and the development of $\mathrm{CKD}^{(5,6)}$. PUFA supplementation was observed as reducing renal inflammation and fibrosis in animal models ${ }^{(6)}$. Recently, an Italian population-based cohort study of 931 adults aged $\geq 65$ years old assessed the association between plasma PUFA levels and renal function. Baseline long-chain $n-3$ and n-6 PUFA were negatively associated with creatinine at the 3-year follow-up. Moreover, participants with higher plasma PUFA at enrollment had a lower risk of developing renal insufficiency (creatinine clearance $<60 \mathrm{ml} / \mathrm{min}$ ) during the 3 -year follow-up ${ }^{(5)}$.

Further epidemiological investigations are needed to confirm the role of PUFA in the renal inflammation and fibrosis pathways of $\mathrm{CKD}^{(6)}$. To the best of our knowledge, no population-based study has examined the association between dietary intakes of PUFA, fish and CKD in the general population. Therefore, we used a large representative,

Abbreviations: AA, arachidonic acid; BMES, Blue Mountains Eye Study; CKD, chronic kidney disease; eGFR, estimated glomerular filtration rate.

*Corresponding author: P. Mitchell, fax +6129845 6117, email paul_mitchell@wmi.usyd.edu.au

† Present address: Centre for Vision Research, University of Sydney, Westmead Hospital, Hawkesbury Road, Westmead, NSW 2145, Australia 
community-based cohort of adults aged $\geq 50$ years to pursue the following aims: (a) to establish whether a cross-sectional association exists between dietary intakes of PUFA and the prevalence of CKD; (b) to determine whether a diet high in fish reduces the likelihood of CKD.

\section{Methods}

\section{Study population}

The present study is based on the second Blue Mountains Eye Study (BMES), a population-based study of participants aged $\geq 49$ years, living in two postcodes of the Blue Mountains region, west of Sydney, Australia, which has studied age-related eye diseases and other health outcomes in an urban Australian population. Details of the study methods have previously been described $^{(7)}$. In brief, following a door-to-door census of the region, 4433 people were identified as eligible for the study. Of these, 3654 participants aged 49 years or older were examined (82.4\% participation; BMES-1) during 1992-4. Surviving baseline participants were invited to attend 5-year follow-up examinations (1997-9, BMES-2), at which 2334 ( $75 \cdot 1 \%$ of survivors) and an additional 1174 newly eligible residents were examined, i.e. those who had become eligible by moving into the area or into the age bracket of the original survey. At the 10-year follow-up (2002-4, BMES-3), 1952 participants $(75.6 \%$ of survivors) were re-examined. The study was approved by the Human Research Ethics Committee of the University of Sydney and was conducted adhering to the tenets of the Declaration of Helsinki. Signed informed consent was obtained from all participants at each examination.

\section{Dietary data}

Dietary data were collected using a 145-item self-administered FFQ, modified for Australian diet and vernacular from an early Willett $\mathrm{FFQ}^{(8)}$, and included reference portion sizes. Participants used a nine-category frequency scale to indicate the usual frequency of consuming individual food items during the past year. The FFQ included details about frequency estimates and fatty acid supplements in order to permit a more detailed analysis of fatty acids.

We extracted separate data on the frequency of consuming fish (any species plus method of preparation) and oily fish (specifically salmon, tuna and sardines). Fish consumption was converted into $\mathrm{g} / \mathrm{d}$ by multiplying the food consumption frequency by fixed portion sizes. The FFQ was validated on seventy-nine participants on three occasions during 1 year by using $4 \mathrm{~d}$ weighed food records ${ }^{(9)}$. The FFQ showed moderate to good agreement for ranking individuals according to their fat intakes (total fat, $r$ 0.68; SFA, $r$ 0.67; MUFA, $r$ 0.54; PUFA, $r$ 0.44) and correctly classified more than $70 \%$ of people within 1 quintile for all types of $\mathrm{fat}^{(9,10)}$.

Dietary intakes were estimated using the Australian Tables of Food Composition (NUTTAB95) ${ }^{(10)}$ and its fatty acid supplement. Additional fatty acid food composition data were added from the Royal Melbourne Institute of Technology database (Melbourne, VIC, Australia) ${ }^{(11)}$, available on
FoodWorks, version 3 (Xyris Software Private Limited, Highgate Hill, QLD, Australia). Long-chain $n$-3 PUFA were calculated from the sum of EPA $(20: 5 n-3)$, docosapentaenoic acid (22:5n-3) and DHA (22:6 n-3). Total $n$-3 PUFA consumption was calculated by adding the intakes of long-chain $n$ - 3 PUFA and $\alpha$-linolenic acid. Total $n$ - 6 PUFA consumption was calculated by adding the intakes of linoleic and arachidonic acid (AA).

\section{Assessment of chronic kidney disease}

Serum creatinine was measured within $4 \mathrm{~h}$ of fasting venous blood collection using a Hitachi 747 biochemistry analyser (Roche Diagnostics, Castle Hill, Sydney, NSW, Australia). Estimated glomerular filtration rate (eGFR) was the preferred measure of kidney function in the present study. Glomerular filtration rate was indirectly estimated using the four-variable Modification of Diet in Renal Disease Study equation ${ }^{(12)}$ :

$$
\begin{aligned}
& \text { Glomerular filtration rate }\left(\mathrm{ml} / \mathrm{min} \text { per } 1.73 \mathrm{~m}^{2}\right) \\
& =186 \times(\text { serum creatinine })^{-1.154} \times(\text { age })^{-0.203} \\
& \times(0.742 \text { if female })(\text { conventional units }) .
\end{aligned}
$$

The main outcome of interest was CKD of stage 3 or greater, defined as an eGFR of $<60 \mathrm{ml} / \mathrm{min}$ per $1.73 \mathrm{~m}^{2}$. For the purposes of the present study, this was defined as CKD of moderate severity.

\section{Collection of other information}

At face-to-face interviews with trained interviewers, a comprehensive medical history that included information about hearing, demographic factors, socio-economic characteristics and lifestyle factors such as smoking was obtained from all participants. History of smoking was defined as never, past or current smoking. Current smokers included those who had stopped smoking within the past year. Alcohol intake was assessed by questions about the frequency of consuming alcoholic drinks (d/week), and consumption was split into three groups (no alcohol, 0-20 and $>20 \mathrm{~g} / \mathrm{d}$ ). Physical inactivity (yes and no) was categorised as answering negatively to have you participated in any recreational exercise/ walk in the last 2 weeks?' Diabetes was defined either from history or by fasting blood glucose $\geq 7.0 \mathrm{mmol} / 1$. Subjects were defined as having hypertension if they had a systolic blood pressure greater than $140 \mathrm{mmHg}$ or a diastolic blood pressure more than $90 \mathrm{mmHg}$ or were on anti-hypertensive medications ${ }^{(13)}$. Serum total homocysteine was determined from blood using the fluorescent polarization immunoassay method on an IMx analyser. BMI was calculated as weight divided by height squared $\left(\mathrm{kg} / \mathrm{m}^{2}\right)$. Serum total cholesterol and TAG were measured on a Reflotron reflectance photometric analyser (Roche Diagnostics).

\section{Statistical analysis}

Analyses including $t$ tests, $\chi^{2}$ tests and logistic regression were performed using the SAS statistical software package version 
9.1 (SAS Institute, Cary, NC, USA). Associations between dietary intakes of PUFA, fish and the prevalence of CKD were examined in logistic regression models, adjusting first for age and sex, and then for confounders that were found to be significantly associated with CKD (i.e. smoking, alcohol intake, BMI, physically inactive, diabetes mellitus, hypertension, serum total homocysteine, serum total cholesterol and serum TAG). Dietary fatty acid intakes were energy adjusted using the residual method described by Willett \& Stampfer ${ }^{(14)}$. Results of the logistic regression analysis are expressed as adjusted OR with 95\% CI. We assessed dietary intakes of PUFA and fish both as categorised (quartiles; with the lowest quartile as the reference group) and continuous (per standard deviation) variables. Statistical significance was defined as $P<0.05$.

\section{Results}

Data for the cross-sectional analyses were obtained from 3508 participants at BMES-2. Of these, 2600 participants had their fasting blood collected at BMES- 2 including serum creatinine measures. In addition, at the same time, these 2600 subjects also had complete dietary data collected at BMES-2. Among these subjects, $504(19.4 \%)$ had CKD of at least stage 3 (moderate CKD) and $2096(80 \cdot 6 \%)$ did not. Table 1 shows the characteristics of participants with and without moderate CKD. Participants with moderate CKD were more likely to be older, be physically inactive and have hypertension, diabetes, stroke, higher $\mathrm{Hb}$, haematocrit and serum homocysteine, total cholesterol and TAG than participants with mild or no CKD. On the other hand, participants with moderate

Table 1. Characteristics of participants with and without chronic kidney disease (CKD)

(Mean values and standard deviations or number of participants and percentages)
CKD were less likely to be male, current smokers and less likely to heavily consume alcohol (Table 1).

Table 2 shows a significant inverse association between long-chain $n$-3 PUFA intake (assessed as a continuous variable) and the prevalence of CKD; each standard deviation increase in energy-adjusted long-chain $n$-3 PUFA intake was associated with a $13 \%$ decrease in the prevalence of CKD (multivariable-adjusted OR $0.87,95 \%$ CI $0.76,0.99$ ). In contrast, each standard deviation increase in energy-adjusted $\alpha$-linolenic acid intake was associated with an $18 \%$ increased likelihood of having CKD. Significant associations were not observed with total n-3 PUFA or total n-6 PUFA intake (Table 2). A non-significant association was observed between fish consumption and moderate CKD (multivariable-adjusted OR $0.89,95 \%$ CI $0 \cdot 78,1.03$ ). We observed a significant linear relationship between dietary intake of $\alpha$-linolenic acid, fish and eGFR (Fig. 1(a) and (b)); however, a linear relationship was not observed with the other dietary PUFA and eGFR (data not shown).

We also analysed the different components of dietary PUFA as quartiles, and observed that participants in the highest quartile of long-chain $n-3$ PUFA intake had a $31 \%$ reduced likelihood of having moderate CKD compared with those in the lowest quartile ( $P$ for trend=0.052; Table 3 ). However, participants in the highest quartile of $\alpha$-linolenic acid intake had an $73 \%$ increased likelihood of having moderate CKD compared with those in the lowest quartile of intake ( $P$ for trend $=0 \cdot 004$ ).

Table 4 shows the association between consumption of fish (in quartiles) and moderate CKD. Participants in the highest quartile of fish consumption had a $32 \%$ reduced likelihood

\begin{tabular}{|c|c|c|c|c|c|c|c|c|c|}
\hline & \multicolumn{4}{|c|}{$\begin{array}{c}\text { Moderate CKD }(\text { eGFR }<60 \mathrm{ml} / \mathrm{min} \\
\left.\text { per } 1.73 \mathrm{~m}^{2}, n 504\right)\end{array}$} & \multicolumn{4}{|c|}{$\begin{array}{c}\text { Without moderate CKD }(\text { eGFR } \geq 60 \mathrm{ml} / \mathrm{min} \\
\text { per } 1.73 \mathrm{~m}^{2}, n \text { 2096) }\end{array}$} & \multirow[b]{2}{*}{$P$} \\
\hline & Mean & SD & $n$ & $\%$ & Mean & SD & $n$ & $\%$ & \\
\hline Age (years) & $73 \cdot 2$ & $8 \cdot 4$ & & & 64.9 & 8.5 & & & $<0.0001$ \\
\hline Male & & & 145 & $28 \cdot 8$ & & & 977 & $46 \cdot 6$ & $<0.0001$ \\
\hline Current smokers & & & 33 & 6.7 & & & 205 & $9 \cdot 9$ & 0.03 \\
\hline \multicolumn{10}{|l|}{ Alcohol consumption (g/d) } \\
\hline No alcohol & & & 137 & $27 \cdot 2$ & & & 380 & $18 \cdot 1$ & $<0.0001$ \\
\hline $0-20$ & & & 310 & $61 \cdot 5$ & & & 1284 & $61 \cdot 3$ & \\
\hline$>20$ & & & 57 & $11 \cdot 3$ & & & 432 & $20 \cdot 6$ & \\
\hline BMl $\left(\mathrm{kg} / \mathrm{m}^{2}\right)$ & $27 \cdot 3$ & 4.6 & & & $27 \cdot 6$ & 4.7 & & & 0.25 \\
\hline Physically inactive & & & 104 & $21 \cdot 8$ & & & 265 & 13.5 & $<0.0001$ \\
\hline Systolic BP (mmHg) & $151 \cdot 8$ & $22 \cdot 4$ & & & $146 \cdot 1$ & 38.6 & & & $<0.0001$ \\
\hline Diastolic BP (mmHg) & 83.9 & $10 \cdot 4$ & & & $86 \cdot 2$ & $36 \cdot 2$ & & & 0.01 \\
\hline Serum total homocysteine $(\mu \mathrm{mol} / \mathrm{l})$ & $15 \cdot 6$ & $6 \cdot 0$ & & & $11 \cdot 2$ & $3 \cdot 8$ & & & $<0.0001$ \\
\hline Serum cholesterol $(\mathrm{mmol} / \mathrm{l})$ & $6 \cdot 0$ & $1 \cdot 1$ & & & $5 \cdot 8$ & $1 \cdot 0$ & & & $<0.0001$ \\
\hline Serum TAG $(\mathrm{mmol} / \mathrm{l})$ & 1.6 & 0.8 & & & 1.5 & 0.9 & & & 0.03 \\
\hline Albumin $(\mathrm{g} / \mathrm{l})$ & 41.5 & $2 \cdot 7$ & & & $41 \cdot 7$ & $2 \cdot 4$ & & & 0.27 \\
\hline $\mathrm{Hb}(\mathrm{g} / \mathrm{l})$ & 143.5 & 13.5 & & & $148 \cdot 4$ & $12 \cdot 1$ & & & $<0.0001$ \\
\hline Haematocrit & 0.43 & 0.04 & & & 0.44 & 0.03 & & & $<0.0001$ \\
\hline Diuretics & & & 103 & $20 \cdot 4$ & & & 148 & $7 \cdot 1$ & $<0.0001$ \\
\hline Hypertension* & & & 323 & $64 \cdot 3$ & & & 1012 & $48 \cdot 4$ & $<0.0001$ \\
\hline Diabetes & & & 71 & $14 \cdot 1$ & & & 198 & 9.5 & 0.002 \\
\hline Stroke $†$ & & & 42 & 8.4 & & & 66 & 3.2 & $<0.0001$ \\
\hline
\end{tabular}

eGFR, estimated glomerular filtration rate (in $\mathrm{ml} / \mathrm{min}$ per $1.73 \mathrm{~m}^{2}$ ) using the four-variable Modification of Diet in Renal Disease Study formula; BP, blood pressure.

* Defined as a systolic BP greater than $140 \mathrm{mmHg}$ or a diastolic BP more than $90 \mathrm{mmHg}$ or using anti-hypertensive medications ${ }^{(13)}$.

$\dagger$ History of previously diagnosed stroke. 
Table 2. Associations between dietary intake of PUFA, fish and the prevalence of chronic kidney disease (CKD) (Mean values, ranges, odds ratios per standard deviation and $95 \%$ confidence intervals)

\begin{tabular}{|c|c|c|c|c|c|c|}
\hline \multirow[b]{3}{*}{ Energy-adjusted fatty acid intake } & \multirow[b]{3}{*}{ Mean } & \multirow[b]{3}{*}{ Range } & \multicolumn{4}{|c|}{ eGFR $<60$ (moderate CKD; $n 504$ (19.4\%)) } \\
\hline & & & \multicolumn{2}{|c|}{ Age-sex adjusted } & \multicolumn{2}{|c|}{ Multivariate adjusted ${ }^{*}$} \\
\hline & & & OR & $95 \% \mathrm{Cl}$ & OR & $95 \% \mathrm{Cl}$ \\
\hline Total $n-3$ PUFA $(1 \mathrm{SD}=0.58)$ & $1 \cdot 10$ & $0.07-5.91$ & $1 \cdot 10$ & $0.99,1.22$ & 1.07 & $0.95,1.20$ \\
\hline Long-chain $n-3$ PUFA (1 SD $=0.30)$ & 0.25 & $0.00-4.44$ & 0.89 & $0.79,1.01$ & 0.87 & $0.76,0.99$ \\
\hline$\alpha$-Linolenic acid $(1 \mathrm{SD}=0.46)$ & 0.84 & $0.07-3.31$ & $1 \cdot 19$ & $1.08,1.32$ & $1 \cdot 18$ & $1.05,1.32$ \\
\hline Total $n-6$ PUFA (1 SD $=4.7)$ & $9 \cdot 0$ & $0.90-39.23$ & 1.08 & $0.97,1.20$ & 1.03 & $0.91,1.17$ \\
\hline Fish $(1 \mathrm{SD}=30.3)$ & $31 \cdot 7$ & $0.00-396.5$ & 0.90 & $0.80,1.01$ & 0.89 & $0.78,1.03$ \\
\hline
\end{tabular}

eGFR, estimated glomerular filtration rate (in $\mathrm{ml} / \mathrm{min}$ per $1.73 \mathrm{~m}^{2}$ ) using the four-variable Modification of Diet in Renal Disease Study formula.

* Adjusted for age, sex, BMI, smoking, alcohol consumption, physical activity, serum total homocysteine, serum total cholesterol, serum TAG, hypertension and history of diagnosed diabetes.

of having an eGFR $<60 \mathrm{ml} / \mathrm{min}$ per $1.73 \mathrm{~m}^{2}$ (multivariableadjusted OR $0 \cdot 68,95 \%$ CI $0 \cdot 48,0 \cdot 97)$. A significant trend was also observed with increasing consumption of fish, i.e. from the first to the fourth quartile ( $P$ for trend=0.001).

We also assessed the association between dietary intake of PUFA and fish and the different eGFR categories, using eGFR $\geq 90 \mathrm{ml} / \mathrm{min}$ per $1.73 \mathrm{~m}^{2}$ as the reference group (Table 5). The association between long-chain $n$-3 PUFA and fish (as continuous variables) was particularly marked in participants with an eGFR of 45 to $<60 \mathrm{ml} / \mathrm{min}$ per $1.73 \mathrm{~m}^{2}$; compared with those with an eGFR of $\geq 90 \mathrm{ml} / \mathrm{min}$ per $1.73 \mathrm{~m}^{2}$ (OR $0.66,95 \%$ CI $0.49,0.89$; OR $0.68,95 \%$ CI $0.53,0.87$,
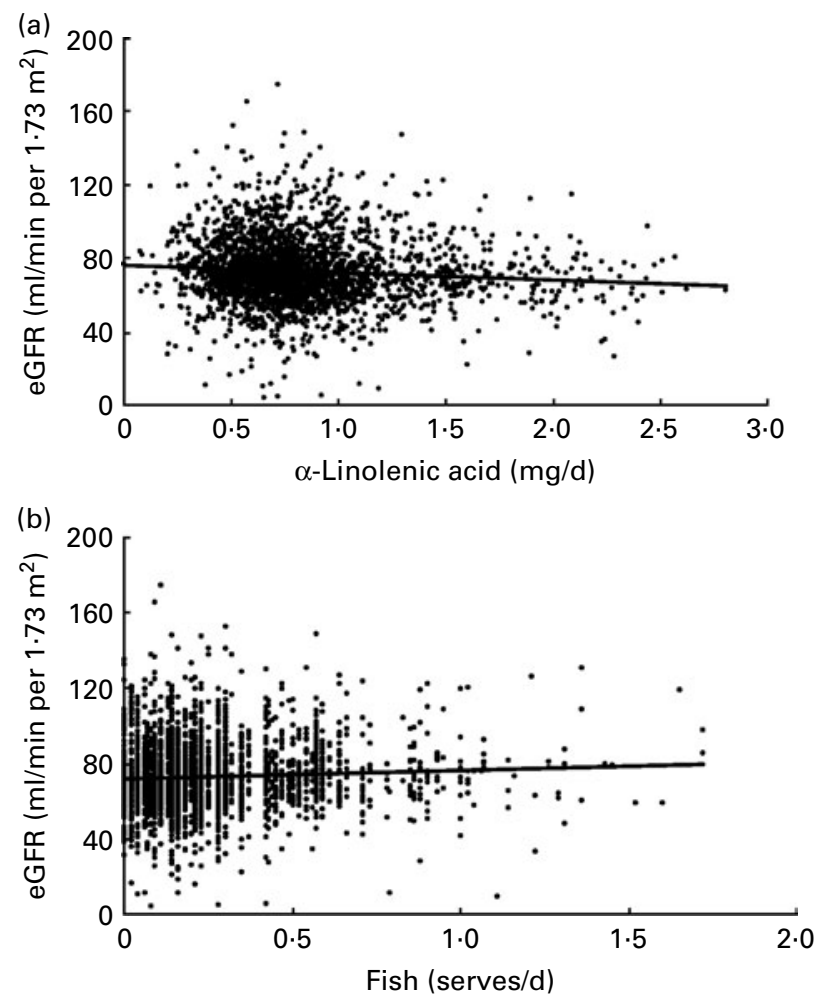

Fig. 1. Linear relationship between dietary intakes of (a) energy-adjusted $\alpha$-linolenic acid (mg/d; $\beta=-3.97, P=0.003$ ) and (b) fish (serves $/ \mathrm{d} ; \beta=4.53$, $P \leq 0.001$ ), with estimated glomerular filtration rate (eGFR) as determined by the four-variable Modification of Diet in Renal Disease Study formula. respectively). Significant positive associations were observed with dietary intake of $\alpha$-linolenic acid in participants with an eGFR of 60 to $<75 \mathrm{ml} / \mathrm{min}$ per $1.73 \mathrm{~m}^{2}$ and an eGFR of 45 to $<60 \mathrm{ml} / \mathrm{min}$ per $1.73 \mathrm{~m}^{2}$. Significant associations with dietary intakes of PUFA and fish were not observed among subjects with an eGFR of $<45 \mathrm{ml} / \mathrm{min}$ per $1.73 \mathrm{~m}^{2}$, possibly due to reduced statistical power.

\section{Discussion}

To the best of our knowledge, the putative association between dietary intakes of PUFA and fish with renal function has not been investigated in a community-based setting. Data from this sample of adults show that increased dietary intake of long-chain $n$-3 PUFA is inversely associated with the prevalence of CKD. Similarly, high consumption of fish reduced the odds of having moderate CKD by $32 \%$. In contrast, a higher intake of $\alpha$-linolenic acid was associated with a $73 \%$ increased likelihood of having prevalent CKD.

The prevalence of moderate CKD in the BMES was 19.4\%, which is relatively similar to the data from the Third National Health and Nutrition Examination Survey ${ }^{(15)}$, which demonstrated a prevalence of $20.6 \%$ in adults aged $\geq 65$ years, and also concurs with a Norwegian study that observed a prevalence of $18.7 \%$ in adults aged 70 years and over ${ }^{(16)}$. The inverse association between dietary intake of long-chain $n-3$ PUFA and the prevalence of CKD concurs with findings of a negative association between plasma $n$-3 PUFA levels and renal function in a cohort of Italians ${ }^{(5)}$. These data are also in agreement with animal models that show that PUFA supplementation reduces the progression of renal disease ${ }^{(6)}$. The underlying mechanism for our observation is unknown. We hypothesise that long-chain n-3 PUFA may protect kidney function by exerting an anti-inflammatory influence through the down-regulation of pro-inflammatory cytokine production, reduction of $\mathrm{NO}$ and expression of endothelial leukocyte adhesion molecules ${ }^{(5,17,18)}$. Furthermore, Cicero et $a l .{ }^{(19)}$ showed that highly purified $n-3$ PUFA long-term supplementation is associated with a significant reduction in systolic and diastolic blood pressure. Hypertension is a risk factor for CKD; hence, the influence of long-chain $n$-3 PUFA on blood pressure may be a potential mechanism by which 
Table 3. Associations between dietary intake of PUFA (in quartiles) and the prevalence of chronic kidney disease (CKD) (Ranges, odds ratios and $95 \%$ confidence intervals)

\begin{tabular}{|c|c|c|c|c|c|}
\hline \multirow{3}{*}{$\begin{array}{l}\text { Energy-adjusted fatty } \\
\text { acid intake (range) }\end{array}$} & \multirow{3}{*}{$\begin{array}{l}\text { No. of cases/no. } \\
\text { at risk }\end{array}$} & \multicolumn{4}{|c|}{ eGFR < 60 (moderate CKD) } \\
\hline & & \multicolumn{2}{|c|}{ Age-sex adjusted } & \multicolumn{2}{|c|}{ Multivariate adjusted ${ }^{*}$} \\
\hline & & OR & $95 \% \mathrm{Cl}$ & OR & $95 \% \mathrm{Cl}$ \\
\hline \multicolumn{6}{|l|}{ Total $n$-3 PUFA } \\
\hline $1(0.04-0.79)$ & $109 / 651$ & $1 \cdot 0$ & Reference & 1.0 & Reference \\
\hline $2(0.79-1.01)$ & $126 / 651$ & 1.33 & $0.97,1.81$ & $1 \cdot 21$ & $0.85,1.72$ \\
\hline $3(1.01-1.29)$ & $131 / 647$ & 1.44 & $1.06,1.96$ & $1 \cdot 29$ & $0.91,1.84$ \\
\hline $4(1 \cdot 29-4 \cdot 76)$ & $138 / 651$ & 1.39 & $1 \cdot 02,1 \cdot 89$ & $1 \cdot 28$ & $0.91,1.81$ \\
\hline$P$ for trend & & \multicolumn{2}{|c|}{0.06} & \multicolumn{2}{|c|}{0.21} \\
\hline \multicolumn{6}{|l|}{ Long-chain $n$-3 PUFA } \\
\hline $1(0.00-0.10)$ & $131 / 651$ & 1.0 & Reference & 1.0 & Reference \\
\hline $2(0.10-0.18)$ & $122 / 649$ & 0.92 & $0.68,1 \cdot 24$ & 0.88 & $0.63,1.24$ \\
\hline $3(0.18-0.31)$ & $141 / 650$ & 1.13 & $0.84,1.51$ & 1.04 & $0.74,1.46$ \\
\hline $4(0.31-4 \cdot 18)$ & $110 / 650$ & 0.77 & $0.56,1.04$ & 0.69 & $0.49,0.99$ \\
\hline$P$ for trend & & \multicolumn{2}{|c|}{0.09} & \multicolumn{2}{|c|}{0.05} \\
\hline \multicolumn{6}{|l|}{$\alpha$-Linolenic acid } \\
\hline $1(0.04-0.59)$ & $92 / 651$ & 1.0 & Reference & 1.0 & Reference \\
\hline $2(0.60-0.76)$ & $130 / 650$ & 1.67 & $1 \cdot 21,2 \cdot 30$ & 1.38 & $0.96,1.99$ \\
\hline $3(0.76-0.99)$ & $134 / 650$ & 1.65 & $1 \cdot 20,2 \cdot 26$ & 1.50 & $1 \cdot 05,2 \cdot 16$ \\
\hline $4(0.99-2.80)$ & $148 / 649$ & 1.93 & $1.41,2.64$ & 1.73 & $1.22,2.47$ \\
\hline$P$ for trend & & \multicolumn{2}{|c|}{0.0003} & \multicolumn{2}{|c|}{0.004} \\
\hline
\end{tabular}

eGFR, estimated glomerular filtration rate (in $\mathrm{ml} / \mathrm{min}$ per $1.73 \mathrm{~m}^{2}$ ) using the four-variable Modification of Diet in Renal Disease Study formula.

* Adjusted for age, sex, BMI, smoking, alcohol consumption, physical activity, serum total homocysteine, serum total cholesterol, serum TAG, hypertension and history of diagnosed diabetes.

it protects the kidneys. However, the association between $n-3$ PUFA and CKD in BMES subjects was independent of the presence of hypertension.

Our finding of a significant negative association between consumption of fish and CKD is novel, but not surprising, given that there is little doubt that long-chain n-3 PUFA in fish are the key nutrients responsible for the health benefits and cardiovascular disease prevention ${ }^{(17,20-22)}$. It has been suggested that regular consumption of fish attenuates inflammatory processes by antagonising pro-inflammatory cytokines that increase the risk of conditions such as cardiovascular disease and possibly $\mathrm{CKD}^{(5,23)}$. Additionally, the hypocholesterolaemic effects of fish proteins have been reported both in animals and humans, together with lower TAG concentrations ${ }^{(24-26)}$. These properties of fish may also account for the observed association with renal function, as hyperlipidaemia is a risk factor for CKD and is thought to cause direct damage to kidney structures and/or increases vascular pathology ${ }^{(1)}$.

Alternatively, the anti-proteinuric effects of $n-3$ PUFA and fish could explain its relationship with moderate CKD, as reductions in proteinuria correlate with the preservation of renal function ${ }^{(27)}$. Previously, Weise et $a l^{(28)}$ used a rat model of membranous nephropathy to demonstrate that rats fed with fish oil had a $25-50 \%$ decline in proteinuria than those fed safflower oil. Hobbs et al. ${ }^{(29)}$ also demonstrated that supplementation of the diet with fish oil prevented the development of proteinuria in hypertensive rats. Additionally, in patients with chronic glomerular disease (an important

Table 4. Associations between dietary intake of fish (in quartiles) and the prevalence of chronic kidney disease (CKD)

(Ranges, odds ratios and $95 \%$ confidence intervals)

\begin{tabular}{|c|c|c|c|c|c|}
\hline \multirow{3}{*}{$\begin{array}{l}\text { Energy-adjusted } \\
\text { fish intake }\end{array}$} & \multirow{3}{*}{$\begin{array}{l}\text { No. of cases/no. } \\
\text { at risk }\end{array}$} & \multicolumn{4}{|c|}{ eGFR $<60$ (moderate CKD) } \\
\hline & & \multicolumn{2}{|c|}{ Age-sex adjusted } & \multicolumn{2}{|c|}{ Multivariate adjusted ${ }^{*}$} \\
\hline & & OR & $95 \% \mathrm{Cl}$ & OR & $95 \% \mathrm{Cl}$ \\
\hline \multicolumn{6}{|l|}{ Fish (g/d) } \\
\hline $1(0.00-14 \cdot 2)$ & $140 / 651$ & 1.0 & Reference & $1 \cdot 0$ & Reference \\
\hline $2(14.2-24 \cdot 3)$ & $134 / 649$ & 1.03 & $0.77,1.38$ & 1.02 & $0.73,1.43$ \\
\hline $3(24.3-39.7)$ & $137 / 650$ & 1.05 & $0.78,1.41$ & 1.04 & $0.74,1.45$ \\
\hline $4(39.8-373.5)$ & $93 / 650$ & 0.64 & $0.47,0.88$ & 0.68 & $0.48,0.97$ \\
\hline$P$ for trend & & \multicolumn{2}{|c|}{0.002} & \multicolumn{2}{|r|}{0.02} \\
\hline
\end{tabular}

eGFR, estimated glomerular filtration rate (in $\mathrm{ml} / \mathrm{min}$ per $1.73 \mathrm{~m}^{2}$ ) using the four-variable Modification of Diet in Renal Disease Study formula.

* Adjusted for age, sex, BMI, smoking, alcohol consumption, physical activity, serum total homocysteine, serum total cholesterol, serum TAG, hypertension and history of diagnosed diabetes. 
Table 5. Associations between dietary intakes of PUFA, fish and the estimated glomerular filtration rate (eGFR) category* (Odds ratios and $95 \%$ confidence intervals)

\begin{tabular}{|c|c|c|c|c|c|c|c|c|}
\hline \multirow[b]{3}{*}{ Energy-adjusted fatty acid intake } & \multicolumn{8}{|c|}{ eGFR category $\dagger$} \\
\hline & \multicolumn{2}{|c|}{$\begin{array}{c}75 \text { to }<90 \\
(n 628,61.5 \%)\end{array}$} & \multicolumn{2}{|c|}{$\begin{array}{c}60 \text { to }<75 \\
(n 1075,73 \cdot 2 \%)\end{array}$} & \multicolumn{2}{|c|}{$\begin{array}{c}45 \text { to }<60 \\
(n 398,50 \cdot 2 \%)\end{array}$} & \multicolumn{2}{|c|}{$<45(n 106,21.2 \%)$} \\
\hline & OR $\ddagger$ & $95 \% \mathrm{Cl}$ & OR $\ddagger$ & $95 \% \mathrm{Cl}$ & OR $\ddagger$ & $95 \% \mathrm{Cl}$ & OR $\ddagger$ & $95 \% \mathrm{Cl}$ \\
\hline Total $n-3$ PUFA $(1 \mathrm{SD}=0.58)$ & 1.07 & $0.92,1.24$ & $1 \cdot 19$ & $1.02,1.40$ & 1.23 & $0.96,1.57$ & $1 \cdot 13$ & $0.73,1.73$ \\
\hline Long-chain $n-3$ PUFA ( 1 SD $=0.30)$ & 0.99 & $0.86,1 \cdot 15$ & 0.99 & $0.85,1.15$ & 0.66 & $0.49,0.89$ & 0.83 & $0.59,1.18$ \\
\hline$\alpha$-Linolenic acid $(1 \mathrm{SD}=0.46)$ & $1 \cdot 10$ & $0.95,1.28$ & 1.25 & $1.07,1.46$ & 1.51 & $1.19,1.91$ & 1.42 & $0.93,2.18$ \\
\hline Total $n-6$ PUFA $(1$ SD $=4.7)$ & 1.03 & $0.90,1.18$ & 1.06 & $0.92,1.23$ & 1.17 & $0.94,1.47$ & 1.06 & $0.69,1.62$ \\
\hline Fish $(1 \mathrm{SD}=30 \cdot 3)$ & 0.94 & $0.83,1.08$ & 0.88 & $0.76,1.01$ & 0.68 & $0.53,0.87$ & 0.69 & $0.46,1.05$ \\
\hline
\end{tabular}

* eGFR ( $\mathrm{ml} / \mathrm{min}$ per $\left.1.73 \mathrm{~m}^{2}\right)$ using the four-variable Modification of Diet in Renal Disease Study formula.

† Compared with participants with eGFR $\geq 90 \mathrm{ml} / \mathrm{min}$ per $1.73 \mathrm{~m}^{2}$ i.e. the reference group.

$\ddagger$ Adjusted for age, sex, BMI, smoking, alcohol consumption, physical activity, serum total homocysteine, serum total cholesterol, serum TAG, hypertension and history of diagnosed diabetes.

cause of CKD), a high-dose $n$-3 PUFA supplementation over 12 weeks reduced proteinuria ${ }^{(30)}$. Finally, Hogg et al. ${ }^{(31)}$. demonstrated that in IgA nephropathy patients, a dosedependent relationship existed between $n$-3 PUFA and reductions in proteinuria.

The role of $\alpha$-linolenic acid on cardiovascular function and cardiometabolic risk remains a matter of debate ${ }^{(32)}$. The positive association between $\alpha$-linolenic acid and moderate CKD observed in the present study was not expected. The specific mechanisms underlying why $n-3$ fatty acids mainly from terrestrial sources and those from mainly marine sources ${ }^{(33)}$ have differing effects on the prevalence of CKD are unclear. One possibility is that $\alpha$-linolenic acid is a plant $n-3$ fatty acid which is poorly converted (less than 5\%) to EPA and DHA, which have been shown to be cardioprotective ${ }^{(34)}$. Thus, increased dietary availability of $\alpha$-linolenic acid does not necessarily enhance DHA synthesis and may even decrease tissue DHA concentrations ${ }^{(35)}$, since DHA synthesis appears to be tightly regulated by feedback inhibition ${ }^{(36)}$. Additionally, while EPA and DHA have numerous antiinflammatory properties, $\alpha$-linolenic acid exerts little influence on inflammatory cytokine production at feasible dietary intakes $^{(18,33,37)}$. Finally, $\alpha$-linolenic acid is present in some vegetable oils and nuts, and animal fats ${ }^{(38)}$; hence, we cannot rule out the possibility that $\alpha$-linolenic acid from both non-animal and animal sources represents a marker of a correlated component of fat or fat-containing food, such as red meat ${ }^{(33)}$, which could lead to, for example, hyperlipidaemia and in turn increase the prevalence of CKD

In the present study, a non-significant increase in the prevalence of moderate CKD was observed with increasing consumption of $n-6$ PUFA. This observation is not surprising as the $n-6$ PUFA AA gives rise to eicosanoid mediators that have established roles in inflammation and AA metabolism, and is a long recognised target for anti-inflammatory therapies ${ }^{(39)}$. The $n-3$ fatty acids EPA and DHA are incorporated into inflammatory cell phospholipids in a time- and dose-dependent manner, and are partly incorporated at the expense of AA. EPA and DHA inhibit AA metabolism. Hence, the production of AA-derived eicosanoids is decreased by $n-3$ PUFA and occurs in a dose-dependent manner ${ }^{(39)}$.
Ageing population trends in Western countries emphasise the need for the identification and development of potential protective strategies for CKD. Hence, the findings presented in this investigation are important as they demonstrate that a diet rich in long-chain $n$-3 PUFA and fish could have a protective influence against the decline in kidney function that is common with ageing ${ }^{(5)}$. Ideally, the diet best designed to preserve renal function would be similar to what has also been proposed for cardiovascular disease, i.e. low in SFA and high in long-chain n-3 PUFA from fish and fish oils, for example, a Mediterranean-style diet ${ }^{(17,40)}$.

Key strengths of the present study include its representative population-based sample with relatively high participation, minimising selection bias, availability of rich covariate/confounder information and the use of standardised protocols for exposure and outcome assessment. However, there are some caveats such as the lack of longitudinal data, which precludes conclusions regarding the prospective nature of the association between dietary intakes of $n-3$ PUFA and fish, and eGFR. Nevertheless, it is unlikely that moderate CKD is an independent predictor of high consumption of PUFA and fish. Second, dietary assessment by the FFQ in which respondents have to estimate typical intake frequencies of food items and their portion sizes can potentially introduce measurement error and bias. We cannot rule this possibility out, but, overall, the validity of fatty acid categories compared with weighed food records was moderately good ${ }^{(9,10,41)}$. Finally, the BMES was not designed to examine the issues of kidney function per se, but rather to examine the issues of sensory loss. Hence, we do not have urinalysis results, although we have estimated renal function in our subjects using a widely accepted method. Nonetheless, limitations of such estimated measures of kidney function exist, specifically serum creatinine is an insensitive marker of glomerular filtration rate in older adults; hence, it is not surprising that the Modification of Diet in Renal Disease formula based predominantly on it is imperfect. The use of this formula cannot circumvent the very significant spectral interferences affecting serum creatinine measurement, e.g. haemolysis ${ }^{(42)}$; as such, eGFR should not be accepted without reservation as a reliable substitute for the direct measurement of renal function. 
In conclusion, higher dietary intake of long-chain n-3 PUFA was associated with a reduced prevalence of moderate CKD. Additionally, increased consumption of fish was associated with a reduced likelihood of CKD in adults aged $\geq 50$ years. These data suggest that long-chain $n$-3 PUFA and fish could have a role in maintaining healthy kidney function, in addition to the roles of these nutrients in the prevention and modulation of other diseases (e.g. autoimmune disorders, coronary heart disease and stroke $)^{(17,18)}$. However, to fully determine the effects of $n-3$ dietary fats and fish on renal function, there is a need for further high-quality population-based studies and randomised controlled trials of long duration.

\section{Acknowledgements}

None of the authors has any conflicts of interest. B. G., D. C. H. and P. M. contributed to the study concept and design. P. M. performed the acquisition of subjects and data. B. G., G. B. and P. M. contributed to the analysis and interpretation of data. B. G. was involved in the preparation of the manuscript. B. G., D. C. H., V. M. F. and P. M. had done the important critical revision. The BMES was supported by the Australian National Health and Medical Research Council (grant no. 974159, 991407, 211069 and 262120).

\section{References}

1. Lauretani F, Maggio M, Pizzarelli F, et al. (2009) Omega-3 and renal function in older adults. Curr Pharm Des 15, 4149-4156.

2. Hamer RA \& El Nahas AM (2006) The burden of chronic kidney disease. BMJ 332, 563-564.

3. Coresh J, Selvin E, Stevens LA, et al. (2007) Prevalence of chronic kidney disease in the United States. JAMA 298, 2038-2047.

4. Shankar A, Wang JJ, Chua B, et al. (2008) Positive association between plasma homocysteine level and chronic kidney disease. Kidney Blood Press Res 31, 55-62.

5. Lauretani F, Semba RD, Bandinelli S, et al. (2008) Plasma polyunsaturated fatty acids and the decline of renal function. Clin Chem 54, 475-481.

6. Baggio B, Musacchio E \& Priante G (2005) Polyunsaturated fatty acids and renal fibrosis: pathophysiologic link and potential clinical implications. J Nephrol 18, 362-367.

7. Attebo K, Mitchell P \& Smith W (1996) Visual acuity and the causes of visual loss in Australia. The Blue Mountains Eye Study. Ophthalmology 103, 357-364.

8. Willett WC, Sampson L, Browne ML, et al. (1988) The use of a self-administered questionnaire to assess diet four years in the past. Am J Epidemiol 127, 188-199.

9. Smith W, Mitchell P, Reay EM, et al. (1998) Validity and reproducibility of a self-administered food frequency questionnaire in older people. Aust $N Z J$ Public Health 22, 456-463.

10. Department of Community Services and Health (1995) NUTTAB95 Nutrient Data Table for Use in Australia. Canberra: Australian Government Publishing Service.

11. Mann NJ, Sinclair AJ, Percival P, et al. (2003) Development of a database of fatty acids in Australian foods. Nutrition $\varepsilon$ Dietetics 60, 42-45.

12. Levey AS, Bosch JP, Lewis JB, et al. (1999) A more accurate method to estimate glomerular filtration rate from serum creatinine: a new prediction equation. Modification of Diet in Renal Disease Study Group. Ann Intern Med 130, 461-470.

13. Whitworth JA, World Health Organization \& International Society of Hypertension Writing Group (2003) 2003 World Health Organization (WHO)/International Society of Hypertension (ISH) statement on management of hypertension. J Hypertens 21, 1983-1992.

14. Willett W \& Stampfer MJ (1986) Total energy intake: implications for epidemiologic analyses. Am J Epidemiol 124, $17-27$.

15. Coresh J, Astor BC, Greene T, et al. (2003) Prevalence of chronic kidney disease and decreased kidney function in the adult US population: Third National Health and Nutrition Examination Survey. Am J Kidney Dis 41, 1-12.

16. Hallan SI, Dahl K, Oien CM, et al. (2006) Screening strategies for chronic kidney disease in the general population: followup of cross sectional health survey. BMJ 333, 1047.

17. Connor WE (2000) Importance of $n-3$ fatty acids in health and disease. Am J Clin Nutr 71, 171S-175S.

18. Calder PC \& Yaqoob P (2009) Omega-3 polyunsaturated fatty acids and human health outcomes. Biofactors 35, 266-272.

19. Cicero AF, Derosa G, Di G, et al. (2010) Omega 3 polyunsaturated fatty acids supplementation and blood pressure levels in hypertriglyceridemic patients with untreated normal-high blood pressure and with or without metabolic syndrome: a retrospective study. Clin Exp Hypertens 32 , $137-144$

20. Wijendran V \& Hayes KC (2004) Dietary $n-6$ and $n-3$ fatty acid balance and cardiovascular health. Annu Rev Nutr 24, 597-615.

21. Hooper L, Thompson RL, Harrison RA, et al. (2006) Risks and benefits of omega 3 fats for mortality, cardiovascular disease, and cancer: systematic review. BMJ 332, 752-760.

22. Hu FB, Bronner L, Willett WC, et al. (2002) Fish and omega-3 fatty acid intake and risk of coronary heart disease in women. JAMA 287, 1815-1821.

23. Din JN, Newby DE \& Flapan AD (2004) Omega 3 fatty acids and cardiovascular disease - fishing for a natural treatment. BMJ 328, 30-35.

24. Gunnarsdottir I, Tomasson H, Kiely M, et al. (2008) Inclusion of fish or fish oil in weight-loss diets for young adults: effects on blood lipids. Int J Obes (Lond) 32, 1105-1112.

25. Demonty I, Deshaies Y, Lamarche B, et al. (2003) Cod protein lowers the hepatic triglyceride secretion rate in rats. J Nutr 133, 1398-1402.

26. Saravanan P, Davidson NC, Schmidt EB, et al. (2010) Cardiovascular effects of marine omega-3 fatty acids. Lancet $\mathbf{3 7 6}$ $540-550$

27. Randomised placebo-controlled trial of effect of ramipril on decline in glomerular filtration rate and risk of terminal renal failure in proteinuric, non-diabetic nephropathy (1997) The GISEN Group (Gruppo Italiano di Studi Epidemiologici in Nefrologia). Lancet 349, 1857-1863.

28. Weise WJ, Natori Y, Levine JS, et al. (1993) Fish oil has protective and therapeutic effects on proteinuria in passive Heymann nephritis. Kidney Int 43, 359-368.

29. Hobbs LM, Rayner TE \& Howe PR (1996) Dietary fish oil prevents the development of renal damage in salt-loaded stroke-prone spontaneously hypertensive rats. Clin Exp Pharmacol Physiol 23, 508-513.

30. De Caterina R, Caprioli R, Giannessi D, et al. (1993) n-3 Fatty acids reduce proteinuria in patients with chronic glomerular disease. Kidney Int 44, 843-850.

31. Hogg RJ, Fitzgibbons L, Atkins C, et al. (2006) Efficacy of omega-3 fatty acids in children and adults with IgA 
nephropathy is dosage- and size-dependent. Clin J Am Soc Nephrol 1, 1167-1172.

32. Brochot A, Guinot M, Auchere D, et al. (2009) Effects of alpha-linolenic acid vs. docosahexaenoic acid supply on the distribution of fatty acids among the rat cardiac subcellular membranes after a short- or long-term dietary exposure. Nutr Metab (Lond) 6, 35.

33. Leitzmann MF, Stampfer MJ, Michaud DS, et al. (2004) Dietary intake of $n-3$ and $n-6$ fatty acids and the risk of prostate cancer. Am J Clin Nutr 80, 204-216.

34. Wang C, Harris WS, Chung M, et al. (2006) n-3 Fatty acids from fish or fish-oil supplements, but not alpha-linolenic acid, benefit cardiovascular disease outcomes in primaryand secondary-prevention studies: a systematic review. $\mathrm{Am}$ J Clin Nutr 84, 5-17.

35. Mantzioris E, James MJ, Gibson RA, et al. (1995) Differences exist in the relationships between dietary linoleic and alphalinolenic acids and their respective long-chain metabolites. Am J Clin Nutr 61, 320-324.
36. Pawlosky RJ, Hibbeln JR, Lin Y, et al. (2003) Effects of beefand fish-based diets on the kinetics of $n-3$ fatty acid metabolism in human subjects. Am J Clin Nutr 77, 565-572.

37. Calder PC (2001) Polyunsaturated fatty acids, inflammation, and immunity. Lipids 36, 1007-1024.

38. Nettleton JA (1991) Omega-3 fatty acids: comparison of plant and seafood sources in human nutrition. J Am Diet Assoc 91, $331-337$.

39. Calder PC (2009) Polyunsaturated fatty acids and inflammatory processes: new twists in an old tale. Biochimie 91, 791-795.

40. de Lorgeril M \& Salen P (2005) Suitability of the Mediterranean-style diet in the modern world. Asia Pac J Clin Nutr 14, S78-S83.

41. Gopinath B, Flood VM, Rochtchina E, et al. (2010) Consumption of omega-3 fatty acids and fish and risk of age-related hearing loss. Am J Clin Nutr 92, 416-421.

42. Lamb EJ, O'Riordan SE \& Delaney MP (2003) Kidney function in older people: pathology, assessment and management. Clin Chim Acta 334, 25-40. 